\title{
Delayed Thoracic Radiation Injury
}

\section{Barry Dicicco}

Inova Fairfax Hospital, Falls Church, USA.

Email: barrydicicco@mac.com

Received July $16^{\text {th }}, 2012$; revised August $26^{\text {th }}, 2012$; accepted September $4^{\text {th }}, 2012$

\begin{abstract}
A case of delayed thoracic radiation injury occurring many years after an initial lung insult is presented. This case involves an individual who developed acute respiratory failure due to severe pleural fibrosis or fibrothorax possibly as a result of distant radiation therapy for Hodgkins disease.
\end{abstract}

Keywords: Radiation Injury; Fibrothorax

\section{Introduction}

Radiation induced lung injury is well known and usually involves either radiation pneumonitis or radiation fibrosis. These changes are most commonly seen within 8 weeks of radiation. It is not known if the effects of radiation can be seen many years after the initial radiation dose and whether or not these changes can be triggered by surgery, trauma or other exogenous insults.

\section{Case Report}

A 70-year-old priest presented to the hospital with severe exertional dyspnea, cough and productive yellow phlegm. An admission chest radiograph (Figure 1) revealed bilateral loculated pleural effusions left greater than right. A chest CT scan (Figure 2) showed bilateral interstitial and airspace opacities, pleural thickening with small effusions but no pulmonary emboli. A sonogram of the legs did not reveal deep venous thrombosis. Prior echocardiograms of the heart documented normal left ventricular function. Initial arterial blood gases were consistent with incipient respiratory failure with a $\mathrm{pO}_{2}$ of 116 $\mathrm{mm} \mathrm{Hg}$, a $\mathrm{pH}$ of 7.35 and a $\mathrm{pCO}_{2}$ of $81 \mathrm{~mm} \mathrm{Hg}$ on $3 \mathrm{li}-$ ters of nasal cannula oxygen. The patient was admitted to a telemetry bed and treated empirically for pneumonia with broad spectrum antibiotics.

Approximately 40 years prior to admission the patient underwent chemotherapy and radiation therapy to the chest for stage IIIB Hodgkins disease and went into complete remission. The patient was asymptomatic until 8 months prior to admission when he developed dyspnea on exertion and was found to have aortic stenosis with a valve area of $1.0 \mathrm{~cm}^{2}$. The aortic valve was trileaflet with extensive valvular and aortic arch calcification consistent with prior radiation damage. A chest radiograph prior to

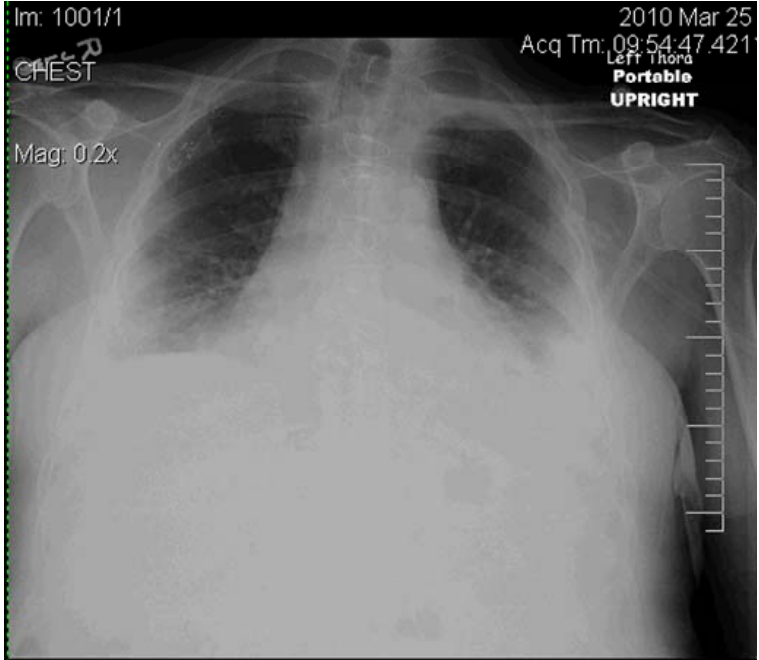

Figure 1. Postoperative CXR showing airspace opacities and pleural thickening.

surgery was unremarkable (Figure 3 ) and a chest CT scan did not reveal significant pleural thickening nor interstitial lung disease (Figure 4). Aortic valve replacement 6 months prior to admission was complicated by a post operative right lower lobe pneumonia without acute lung injury, post operative atrial fibrillation and cardiac tamponade requiring a pericardial window. An arterial blood gas on postoperative day \#7 on 6 liters of nasal cannula oxygen showed a $\mathrm{paO}_{2}$ of $82 \mathrm{~mm} \mathrm{Hg}$, a pH of 7.36 and a $\mathrm{pCO}_{2}$ of $32 \mathrm{~mm} \mathrm{Hg}$.

Following discharge from the hospital after aortic valve replacement, the patient's dyspnea progressively worsened and he became oxygen dependent. Pulmonary function studies showed a forced vital capacity of 1.07 liters (26\% predicted) and an FEV1\% of $70 \%$. Pre-operatively the $\mathrm{FVC}$ was 2.63 liters (62\% predicted) with a total lung 


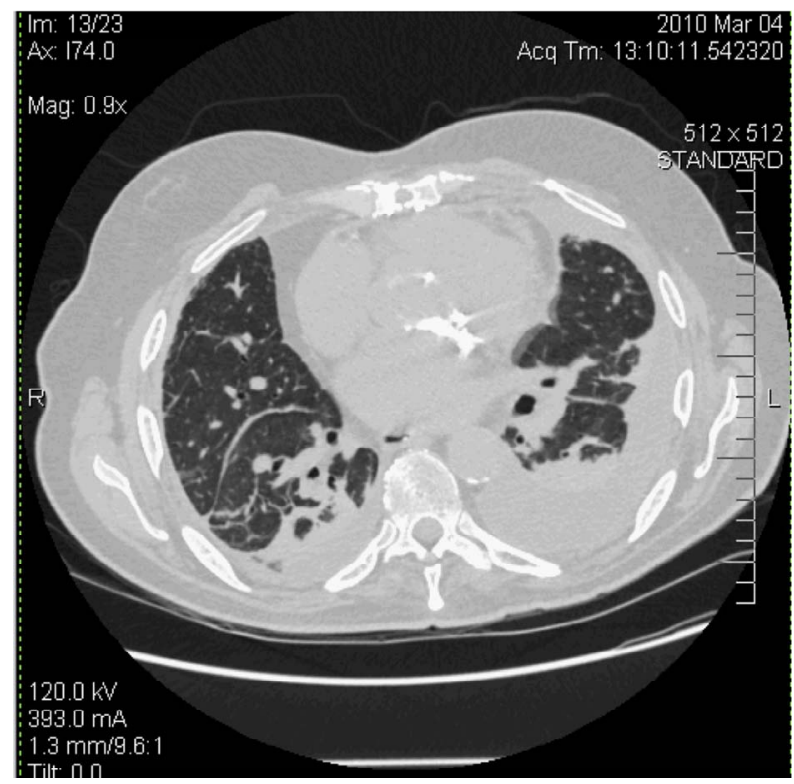

Figure 2. Postoperative chest CT.

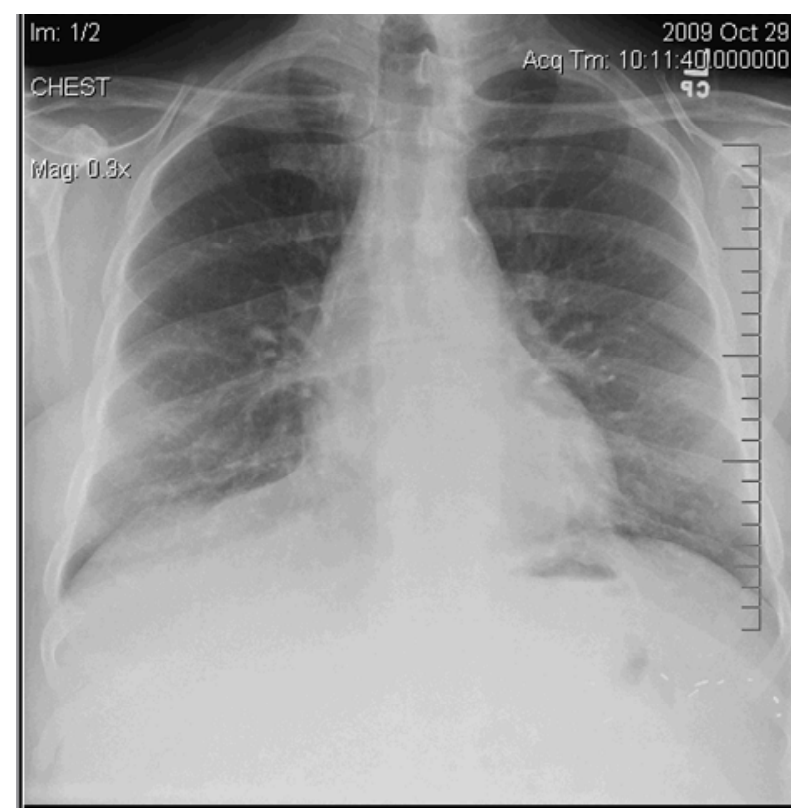

Figure 3. Preoperative CXR.

capacity of 4.71 liters ( $70 \%$ predicted). Amiodarone treatment for atrial fibrillation continued for 4 months post operatively, however a chest CT scan did not show significant interstitial lung disease at that time. Fluoroscopy of both hemidiaphragms documented movement. An echocardiogram 6 weeks following surgery revealed an ejection fraction of $55 \%$, good aortic valve function and a peak gradient of $25 \mathrm{~mm}$, mild mitral regurgitation and an estimated pulmonary artery pressure of $45 \mathrm{~mm}$. Outpatient thoracentesis did not alleviate the patient's symptoms and the analysis included a protein of 2.7 (serum 5.1), a LDH of 1182, $784 \mathrm{WBCs}$ and 127,000 RBCs.

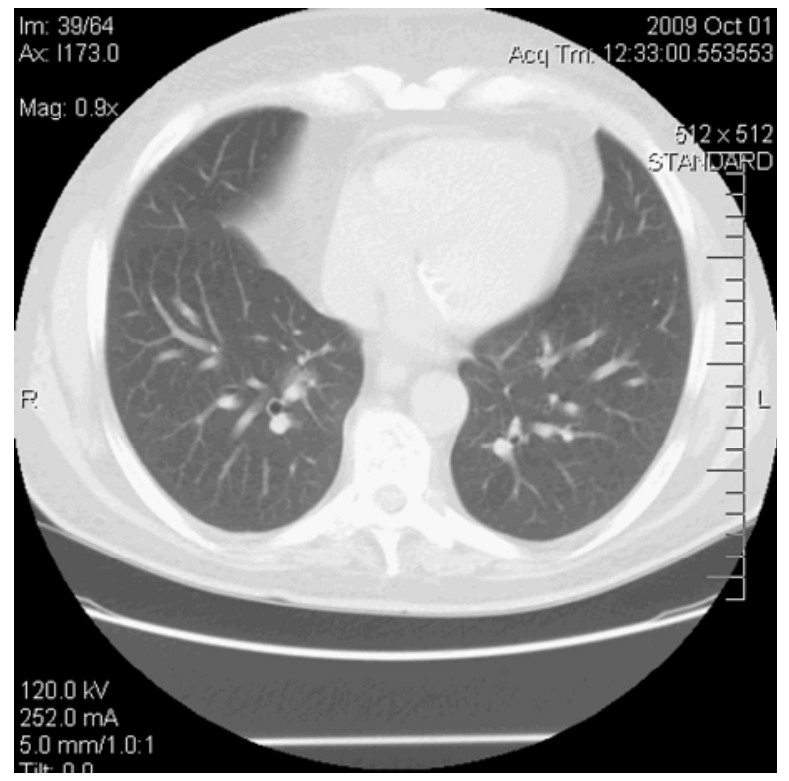

Figure 4. Preoperative Chest CT.

The patient's current hospital course was notable for rapid deterioration and transfer to the intensive care unit for intubation and mechanical ventilatory support after an arterial blood gas showed a $\mathrm{pH}$ of 7.16 and a $\mathrm{pCO}_{2}$ of $116 \mathrm{~mm} \mathrm{Hg}$ on 4 liters of nasal cannula oxygen. Laboratory testing included a WBC count $10,960 \mu \mathrm{L}$, a BUN of $40 \mathrm{mg} / \mathrm{dL}$, creatinine of $1.2 \mathrm{mg} / \mathrm{dL}$ and negative serial troponins. A chest CT scan confirmed consolidation of both lower lobes consistent with pneumonia. Hypotension developed requiring vasopressors. A right heart catheterization revealed a right atrial pressure of 12 $\mathrm{mmHg}$, a right ventricular pressure of $48 / 2 \mathrm{mmHg}$, a pulmonary artery pressure of $36 / 12 \mathrm{mmHg}$ (mean 19 $\mathrm{mmHg}$ ), a PCWP of $16 \mathrm{mmHg}$, and a cardiac output of 4.9 liters per minute. Broad spectrum antibiotics and diuretics did not improve the patient's condition and weaning from the ventilator was unsuccessful. After a thorough discussion with the patient's family, the patient was removed from the ventilator after 12 days and expired.

A post mortem examination revealed diffuse bilateral pleural fibrosis (see Figure 5), dense mediastinal fibrosis, organizing pneumonia and interstitial pulmonary fibrosis. There was also fibrotic obliteration of the pericardial cavity.

\section{Discussion}

Radiation induced lung injury is well known and usually involves either radiation pneumonitis or radiation fibrosis. These changes are dose related and usually apparent within 8 weeks of radiation. On occasion fibrotic changes in the lungs can progress over years and even the late appearance of bronchiolitis obliterans organizing pneumonia has been described [1]. Small pleural effusions 


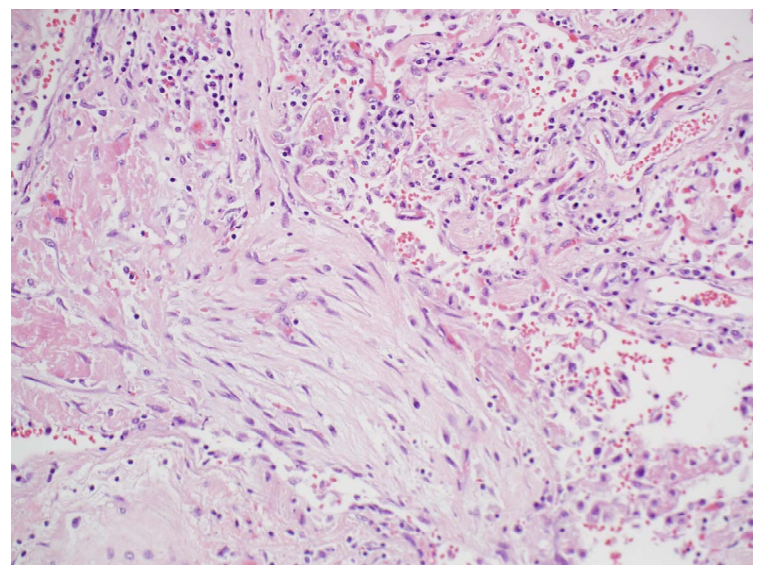

Figure 5. Photomicrograph of pleura showing diffuse fibrosis.

may occur during the initial phase of radiation injury, but chronic pleural disease with the development of pleural fibrosis or fibrothorax has not been described. It also well known that radiation can cause cardiac disease including pericarditis, valvular thickening and calcification with insufficiency, myocardial fibrosis with congestive heart failure or conduction abnormalities such as left bundle branch block or complete heart block [2]. Accelerated atherosclerosis has also been described due to intimal proliferation of fibrous tissue [3].

This patient manifested no clinical symptoms of radiation induced lung injury at the time of radiation 40 years prior to aortic valve surgery and was asymptomatic for many decades following. The striking feature of this case is the development of severe restrictive ventilatory impairment following aortic valve surgery. Post operative pulmonary function studies revealed a marked decline in lung volumes associated with severe exertional dyspnea and oxygen dependence. Although the post operative course was complicated by pneumonia, there was no clinical nor radiographic evidence of acute lung injury and no significant interstitial lung disease until a few weeks before death. Although it is possible that the patient received bleomycin 40 years earlier and that perioperative oxygen therapy may have caused the interstitial pulmonary fibrosis noted on autopsy, it is unlikely that this would explain the extensive pleural fibrosis and restricted chest physiology following surgery. Furthermore, the overall degree of pericardial fibrosis found on autopsy exceeded that which is normally found following heart surgery even in the presence of tamponade. Chest radiographs and $\mathrm{CT}$ scans revealed pleural thickening with a marked reduction in lung volumes. The clinical course was compatible with progressive ventilatory respiratory failure. Pericardial disease was excluded as a contributing factor based on clinical data including a right heart catheterization.

Pulmonary fibrotic changes are believed to be medi- ated by a number of different cytokines [4] Transforming growth factor beta 1 (TGF-beta) is known to be up regulated following lung radiation and this cytokine can induce fibroblast collagen deposition [5].

Other cytokines such as tumor necrosis factor-alpha (TNFa), interleukin 1-alpha (IL-1a), platelet derived growth factor and fibroblast growth factor are all up regulated as well and can cause inflammation and subsequent pulmonary fibrosis. What is not known is how long this up regulation persists and whether or not this process may be accelerated by surgery, trauma or other outside stimuli.

The post mortem examination revealed fibrotic changes in the pleura, pericardium and lung and gross examination of the lungs was consistent with a fibrothorax. Enhanced fibrotic responses to surgery are well known in the abdomen with subsequent development of adhesions and bowel obstruction. Further studies are suggested to determine if cardiothoracic surgery has the potential to induce similar changes in the setting of prior radiation injury with the development of pleural, pericardial and pulmonary fibrosis.

\section{Conclusion}

Radiation therapy has the potential to cause pulmonary fibrosis and/or pneumonitis acutely. We present a case of delayed thoracic radiation injury possibly caused by the effects of cardiothoracic surgery and prior radiation therapy for Hodgkins disease with resultant acute respiratory failure due to pleural fibrosis and severe restrictive ventilatory respiratory failure.

\section{REFERENCES}

[1] N. Takigawa, Y. Segawa, T. Saeki, et al., "Bronchiolits Obliterans Organizing Pneumonia Syndrome in BreastConserving Therapy for Early Breast Cancer: Radiation-Induced Lung Toxicity," International Journal of Radiation Oncology Biology Physics, Vol. 48, No. 3, 2000, pp. 751-755. doi:10.1016/S0360-3016(00)00654-4

[2] S. L. Hancock, M. A. Tucker and R. T. Hoppe, "Factors Affecting Late Mortality from Heart Disease after Treatment of Hodgkin's Disease," Journal of the American Medical Association, Vol. 270, No. 16, 1993, pp. 19491956. doi:10.1001/jama.270.16.1949

[3] D. Reber, et al., "Heart Diseases Following Mediastinal Irradiation: Surgical Management," European Journal of Cardiothoracic Surgery, Vol. 9, No. 4, 1995, pp. 202-205. doi:10.1016/S1010-7940(05)80145-9

[4] G. W. Morgan, B. Pharm and S. N. Breit, "Radiation and the Lung: A Reevaluation of the Mechanisms Mediating Pulmonary Injury," International Journal of Radiation Oncology Biology Physics, Vol. 31, No. 2, 1995, pp. 361369. doi:10.1016/0360-3016(94)00477-3

[5] J. A. Lasky and W. W. Merril, "Radiation Induced Lung Injury," Uptodate, 2006. 